\title{
SPACES WITH A COMPACT LIE GROUP OF TRANSFORMATIONS
}

\section{A. M. GLEASON}

Introduction. A topological group $B$ is said to act on a topological space $R$ if the elements of $B$ are homeomorphisms of $R$ onto itself and if the mapping $(\sigma, p) \rightarrow \sigma(p)$ of $\leftrightarrow \times R$ onto $R$ is continuous. Familiar examples include the rotation group acting on the Cartesian plane and the Euclidean group acting on Euclidean space. The set $\mathbb{S}(p)$ (that is, the set of all $\sigma(p)$ where $\sigma \in(S)$ is called the orbit of $p$. If $p$ and $q$ are two points of $\mathbb{R}$, then $\mathcal{S}(p)$ and $\mathcal{S}(q)$ are either identical or disjoint, hence $R$ is partitioned by the orbits. The topological structure of the partition becomes an interesting question. In the case of the rotations of the Cartesian plane we find that, excising the singularity at the origin, the remainder of space is fibered as a direct product. A similar result is easily established for a compact Lie group acting analytically on an analytic manifold. In this paper we make use of Haar measure to extend this result to the case of a compact Lie group acting on any completely regular space. The exact theorem is given in $\$ 3$. $\$ \$ 1$ and 2 are preliminaries, while in $\$ 4$ we apply the main result to the study of the structure of topological groups. These applications form the principal motivation of the entire study, ${ }^{1}$ and the author hopes to develop them in greater detail in a subsequent paper.

1. An extension theorem. In this section we shall prove an extension theorem which is an elementary generalization of the well known theorem of Tietze. For the sake of completeness we include the proof although it proceeds along standard lines.

We recall that a topological space $R$ is said to be completely regular if it is a Hausdorff space and if, for any closed set $F$ and point $p \notin F$, there exists a continuous, real-valued function $f$ defined on $R$ such that $f(p)=1$ and $f(q)=0$ if $q \in F$. If we can choose such a function, we can choose another which is also non-negative; for example, let $h(q)$ $=\max (0, f(q))$.

\subsection{Lemma. Let $F$ be a closed subset of a completely regular space $R$.}

Presented to the Society, September 9, 1948; received by the editors October 21, 1948.

1 The author began this study thinking oniy in terms of coset decompositions of topological groups. He is indebted to Professor Deane Montgomery for suggesting Theorem 3.4. 
Let $C$ be a compact subset of $R$ disjoint from $F$. Let $\lambda$ be a positive real number. Then there exists a continuous, real-valued function $g$ defined on R such that:

(1) $0 \leqq g(q) \leqq \lambda$ for all $q \in R$.

(2) $g(q)=0$ if $q \in F$.

(3) $g(q)=\lambda$ if $q \in C$.

Proof. For each $p \in C$ we choose a continuous non-negative function $f_{p}$ such that $f_{p}(p)=1$ and $f_{p}(q)=0$ if $q \in F$. Let $V(p)=\hat{q}\left(f_{p}(q)\right.$ $>1 / 2)$. Since $f_{p}$ is continuous, $V(p)$ is an open neighborhood of $p$; hence $C \subset \cup_{p \in c} V(p)$. $C$, being compact, is covered by a finite subcollection of the $V(p)$, say $C \subset U_{i=1}^{n} V\left(p_{i}\right)$. Define $g(q)=\min \left(\lambda, 2 \lambda \sum_{i=1}^{n} f_{p_{i}}(q)\right)$. The function $g$ is easily verified to have the required properties.

1.2 Definition. A real-valued function $f$ is said to vanish at infinity if, for any $\epsilon>0$, there is a compact set $C$ such that $|f(p)|<\epsilon$ for all $p \notin C$.

The functions which vanish at infinity form a linear class.

1.3 Lemma. Let $D$ be a closed subset of a completely regular space $R$. Let $f$ be a continuous, non-negative function defined on $D$ and vanishing at infinity such that $f(p) \leqq k$ for all $p \in D$. Then there exists a function $g$ defined on $R$ such that;

(1) $0 \leqq g(p) \leqq k / 3$ for all $p \in R$.

(2) $0 \leqq f(p)-g(p) \leqq 2 k / 3$ for all $p \in D$.

(3) $g$ vanishes at infinity on $D$.

Proof. We assume that $k>0$, otherwise the lemma is trivial. Let $C=\hat{p}(p \in D, f(p) \geqq 2 k / 3)$ and $F=\hat{p}(p \in D, f(p) \leqq k / 3)$. These sets are closed. Since $f$ vanishes at infinity the sets $C$ and $\mathrm{Cl}(D-F)$ are compact. We apply Lemma 1.1 to the sets $C$ and $F$, choosing $\lambda=k / 3$. We obtain a function $g$ which satisfies our requirements. For $g$ vanishes on all of $D$ except the compact set $\mathrm{Cl}(D-F)$, and the inequalities of (2) follow from

$$
\begin{aligned}
& 2 k / 3 \leqq f(p) \leqq k, \quad g(p)=k / 3 \quad \text { for } p \in C, \\
& k / 3 \leqq f(p) \leqq 2 k / 3, \quad 0 \leqq g(p) \leqq k / 3 \quad \text { for } p \in D-C-F, \\
& 0 \leqq f(p) \leqq k / 3, \quad g(p)=0 \quad \text { for } p \in F .
\end{aligned}
$$

1.4 THEOREM. Let $D$ be a closed subset of a completely regular space R. Let $f$ be a continuous, real-valued function defined on $D$ and vanishing at infinity such that $|f(p)| \leqq k$ for all $p \in D$. Then there exists a continuous, real-valued function $h$ defined on $R$ such that $|h(p)| \leqq k$ for all $p \in R$ and $h(p)=f(p)$ for all $p \in D$. 
Proof. We assume to begin with that $f$ is non-negative. We apply the preceding lemma to $f$ and obtain a function $g_{1}$ defined on $R$ and vanishing at infinity on $D$ such that $0 \leqq g_{1}(p) \leqq k / 3$ for all $p \in R$ and $0 \leqq f(p)-g_{1}(p) \leqq 2 k / 3$ for all $p \in D$. Since the function $f-g_{1}$ is defined and vanishes at infinity on $D$ we can apply the lemma again and obtain a function $g_{2}$ defined on $R$ and vanishing at infinity on $D$ such that $0 \leqq g_{2}(p) \leqq 2 k / 3^{2}$ for all $p \in R$ and $0 \leqq f(p)-g_{1}(p)-g_{2}(p)$ $\leqq 2^{2} k / 3^{2}$ for all $p \in D$. Applying the lemma repeatedly, we obtain a sequence of functions $\left\{g_{n}\right\}$ each defined on $R$ and vanishing at infinity on $D$ such that:

(1) $0 \leqq g_{n}(p) \leqq 2^{n-1} k / 3^{n}$ for all $p \in R$.

(2) $0 \leqq f(p)-\sum_{i=1}^{n} g_{i}(p) \leqq 2^{n} k / 3^{n}$ for all $p \in D$.

Put $h(p)=\sum_{i=1}^{\infty} g_{i}(p)$. From (1) it follows that the series is uniformly convergent and, therefore, that $h$ is continuous; moreover, $h(p) \leqq k$ for all $p \in R$. It follows from (2) that $h(p)=f(p)$ for all $p \in D$. This completes the proof in the case that $f$ is non-negative. We note that in this case the extended function turned out to be non-negative.

In the general case we put $f_{1}(p)=\max (0, f(p))$ and $f_{2}(p)$ $=\max (0,-f(p))$. Then $f_{1}$ and $f_{2}$ are non-negative functions which vanish at infinity on $D$ with the same bound as $f$; furthermore, $f=f_{1}-f_{2}$. Let $h_{1}$ and $h_{2}$ be non-negative extensions of $f_{1}$ and $f_{2}$ respectively, obtained as above. Then $h=h_{1}-h_{2}$ is the required extension of $f$.

In what follows we shall not need the full force of this theorem, but only the fact that we can extend any function from a compact set to the whole of $R$.

2. Topological results. In this section we study the action of a topological group on a topological space without any assumptions of an analytic character. Theorem 2.3 is a useful criterion for determining if the orbit partition is indeed a direct product fibering.

2.1 Lemma. Let \&s be a topological group which acts on a topological space $R$. If $C$ is a compact subset of $\mathbb{S}$ and $F$ is a closed subset of $R$, then $C(F)$ is closed.

Proof. Suppose $p \notin C(F)$. We must show that there is a neighborhood $V$ of $p$ such that $V \subset R-C(F)$. For each $\sigma \in C, \sigma^{-1}(p) \in R-F$. Because the latter set is open, we can choose neighborhoods $U_{\sigma}$ of $\sigma^{-1}$ and $V_{\sigma}$ of $p$ such that $U_{\sigma}\left(V_{\sigma}\right) \subset R-F$. $C^{-1}$, being compact, is covered by the union of a finite subcollection of the $U_{\sigma}$, say $C^{-1} \subset U_{i=1}^{n} U_{\sigma_{i}}$. Put $V=\bigcap_{i=1}^{n} V_{\sigma_{i}} . C$ is a neighborhood of $p$. Now $U_{\sigma_{i}}(V) \subset U_{\sigma_{i}}\left(V_{\sigma_{1}}\right) \subset R-F$; hence $C^{-1}(V) \subset U_{i=1}^{n} U_{\sigma_{i}}(V) \subset R-F$, from which it follows that $V \subset R-C(F)$. 
2.2 LEMMA. Let (S) be a topological group which acts on a topological space $R$. If $C$ is a compact subset of $(S)$ and $E$ is any subset of $R$, then $C(\mathrm{Cl}(E))=\mathrm{Cl}(C(E))$.

Proof. For any element $\sigma \in C$ we have $\sigma(E) \subset C(E)$, hence $\mathrm{Cl}(\sigma(E)) \subset \mathrm{Cl}(C(E))$. Since $\sigma$ is a homeomorphism, $\mathrm{Cl}(\sigma(E))$ $=\sigma(\mathrm{Cl}(E))$, hence $C(\mathrm{Cl}(E))=\mathrm{U}_{\sigma \in C} \sigma(\mathrm{Cl}(E)) \subset \mathrm{Cl}(C(E))$. On the other hand, $C(E) \subset C(\mathrm{Cl}(E))$ and, since the latter set is closed by the preceding lemma, $\mathrm{Cl}(C(E)) \subset C(\mathrm{Cl}(E))$.

In what follows we shall denote by $\$_{p}$ the set of all elements of $B$ which leave the point $p$ fixed. Evidently $\mathbb{S}_{p}$ is a closed subgroup of $(\$)$.

2.3 THEOREM. Let (S) be a compact topological group which acts on a topological space $R$. Let $\Omega$ be a closed subgroup of $\$$. Let $C$ be a closed subset of $R$ such that if $p \in C$ then $\mathbb{S}_{p}=\Omega$ and $\mathbb{S}(p) \cap C=\{p\}$. Then the mapping $(\sigma \Omega, p) \rightarrow \sigma(p)$ of $(\$ / \Omega) \times C$ onto $B(C)$ is a homeomorphism.

Proof. It is clear that the mapping $(\sigma \Omega, p) \rightarrow \sigma(p)$ where $p \in C$ is well-defined. The continuity of this mapping follows from the continuity of $(\sigma, p) \rightarrow \sigma(p)$ and the definition of the topology in $(\& / \Omega$. Suppose that $\sigma(p)=\tau(q)$ where $p, q \in C$. Then $\tau^{-1} \sigma(p)=q$ and it follows that $p=q$ and $\tau^{-1} \sigma \in \Omega ; \tau$ and $\sigma$ determine the same left coset, and hence the mapping is one-to-one. It remains to show that it carries open sets into open sets. For this purpose we may confine ourselves to open sets of the type $U \times V$ where $U$ and $V$ are open sets of $(3 / \Omega$ and $C$ respectively. Since the mapping is one-to-one we need only show that the complements of such sets are carried into closed sets. The complement of $U \times V$ is $(((B / \Omega)-U) \times C) \cup((B / \Omega)$ $\times(C-V))$, and its image is $\left.(\$)-U_{1}\right)(C) \cup(S)(C-V)$ where $U_{1}$ is the complete inverse image of $U$ under the natural map of $\&$ onto $B / \Omega$. By Lemma 2.1 this image is closed and the theorem is proved.

3. Principal results. We now consider the case of a compact Lie group acting on a completely regular space. Beginning with the simplest case in which we are aided by the existence of analytic coordinates, we show that it is possible to construct local "cross-sections"; that is, closed sets which meet the orbit of each nearby point just once. The importance of these sets is reflected in Theorem 2.3. The principal weapon is the use of Haar measure to produce an "orbitfaithful" representation of the group acting on the space.

3.1 LemMA. Let (5) be a closed subgroup of a Lie group $\mathfrak{M}$. Then there exists a compact neighborhood $N$ of the identity in $\mathfrak{M}$ and a closed subset $C$ of $N$ such that every right coset of (5) which intersects $N$ has exactly one point in common with $C$. 
Since this result is standard in the theory of Lie groups, the proof will not be given here. A proof can be found in Chevalley, Theory of Lie groups: I, Princeton University Press, 1946, pp. 109-110.'

3.2 LemMA. Let \&s be a compact group of $n \times n$ matrices. We assume that (\&) acts on the Cartesian space of all $n \times n$ matrices by matrix multiplication. Then there exists a closed neighborhood $N$ of the identity matrix and a closed subset $C$ of $N$ each that the orbit of every point of $N$ has exactly one point in common with $C$. We can choose $N$ so that it contains only nonsingular matrices.

Proof. The Cartesian space $S$ of all $n \times n$ matrices contains the group $\mathfrak{M}$ of all nonsingular $n \times n$ matrices as an open subspace. (S) is a subgroup of $\mathfrak{M}$, and the orbits under $\mathbb{B S}$ of points in $\mathfrak{M}$ are just the right cosets of $\mathbb{B}$ in $\mathfrak{M}$. Being compact, $\mathbb{B}$ is a closed subgroup of $\mathfrak{M}$, which is, of course, a Lie group. We choose the sets $N$ and $C$ as in Lemma 3.1. Then $N$ is a closed neighborhood of the identity matrix in $S$ which contains only nonsingular matrices and $C$ has exactly one point in common with the orbit of each point of $N$.

3.3 TheOREM. Let $\$ 3$ be a compact Lie group which acts on a completely regular space $R$. Let $p$ be a point of $R$ such that $\sigma(p) \neq p$ unless $\sigma$ is the identity. Then there exists a closed neighborhood $N$ of $p$ and a closed subset $C$ of $N$ such that the orbit of every point of $N$ has exactly one point in common with $C$.

PROOF. Let $H$ be a faithful representation of $\mathbb{B}$ as a group of $n \times n$ matrices. ${ }^{3}$ We shall define a function $J$ on $R$ with values in the space of all $n \times n$ matrices, that is to say, $n^{2}$-dimensional Cartesian space. On the orbit of $p$ let $J(\sigma(p))=H(\sigma)$ (the condition that $\sigma(p) \neq p$ unless $\sigma$ is the identity insures against inconsistency) and let $J$ be extended to the rest of $R$ in any continuous fashion. This extension is possible by virtue of Theorem 1.4 and the fact that $\$(p)$, as the image of a compact set, is compact. Define a new matrix valued function $K$ by setting

$$
K(q)=\int_{\odot} H\left(\tau^{-1}\right) J(\tau(q)) d \tau,
$$

where the integration is with respect to the Haar measure of $\mathbb{G}$. The

\footnotetext{
2 Our statement of the lemma does not agree with Chevalley's Proposition 1, but the proof of the latter contains a complete proof of Lemma 3.1.

3 The Peter-Weyl theorem assures us of the existence of a faithful matrix representation (cf. Pontrjagin, Topological groups, Princeton University Press, 1939, pp. 87-125).
} 
hypothesis assures that $\tau(q)$ is a continuous function of $\tau$ and $q$ together, whence it follows easily that $K$ is continuous. The important relation $K(\sigma(q))=H(\sigma) K(q)$ follows from the invariant property of the integration, for

$$
\begin{aligned}
K(\sigma(q)) & =\int_{\Theta} H\left(\tau^{-1}\right) J(\tau \sigma(q)) d \tau=\int_{\Theta} H\left(\sigma \rho^{-1}\right) J(\rho(q)) d \rho \\
& =H(\sigma) \int_{\Theta} H\left(\rho^{-1}\right) J(\rho(q)) d \rho=H(\sigma) K(q) .
\end{aligned}
$$

Moreover, $K(p)=\int_{\Theta} H\left(\tau^{-1}\right) H(\tau) d \tau=I$, the identity matrix.

The group (S) acts by matrix multiplication on the space of all $n \times n$ matrices through the medium of the representation $H$. By Lemma 3.2 there is a closed neighborhood $N_{1}$ of the identity matrix and a closed subset $C_{1}$ of $N_{1}$ such that if $M \in N_{1}$ there is exactly one point of the form $H(\sigma) M$ which is in $C_{1}$. We may assume that $N_{1}$ contains only nonsingular matrices. Let $N=K^{-1}\left(N_{1}\right)$ (the complete inverse image of $N_{1}$ under $K$ ) and $C=K^{-1}\left(C_{1}\right)$. Then $N$ is a closed neighborhood of $p$ and $C$ is a closed subset of $N$.

Suppose now that $q \in N$. Then $K(q) \in N_{1}$ and there is exactly one point of $C_{1}$ of the form $H(\sigma) K(q)$. Since $K(q)$ is nonsingular it can have only one representation in this form. Thus there is exactly one element $\sigma \in \mathbb{S}$ such that $K(\sigma(q))=H(\sigma) K(q) \in C_{1}$; that is, there is exactly one element $\sigma \in B$ such that $\sigma(q) \in C$. This completes the proof.

3.4 THEOREM. Let \& be a compact Lie group which acts on a completely regular space $R$. Let $p$ be a point of $R$ and suppose that $\mathbb{S}_{q}$ is conjugate to $\mathbb{G}_{p}$ for all $q \in R$. Then there exists a closed neighborhood $N$ of $p$ and a closed subset $C$ of $N$ such that the orbit of every point of $N$ has exactly one point in common with $C$.

Proof. Let $S$ be the subset of $R$ consisting of all points left fixed by every element of $\mathbb{S}_{p} . S$ is closed. If $s \in S$, then $\mathbb{S}_{s} \supset \mathbb{S}_{p}$, but in a compact Lie group no subgroup contains one of its conjugates properly, hence $\mathbb{S}_{s}=\mathbb{S}_{p}$. If $q \in R, \mathbb{S}_{q}=\sigma \mathbb{S}_{p} \sigma^{-1}$ for some $\sigma \in\left(\mathcal{S}\right.$. Then $\mathbb{S}_{\sigma^{-1}(q)}$ $=\sigma^{-1} \mathscr{S}_{q} \sigma=\mathscr{S}_{p}$; hence $\sigma^{-1}(q) \in S$ and $q \in \circlearrowleft(S)$. We have proved that $\mathbb{S}(S)=\mathbb{R}$; moreover, for any set $F \subset \mathbb{R}, \mathbb{S}(F)=\mathbb{S}(S \cap \mathbb{S}(F))$.

Let $\mathfrak{S}$ be the normalizer of $\mathcal{G}_{p}$ in $\mathbb{S}$. $\mathfrak{W}$ is a closed subgroup of $\mathbb{B}$. $\mathfrak{S}$ maps $S$ into itself, for, if $\sigma \in \mathfrak{W}$ and $s \in S$, then $\mathcal{G}_{\sigma(s)}=\sigma \mathcal{G}_{s} \sigma^{-1}$ $=\sigma \mathfrak{S}_{p} \sigma^{-1}=\mathfrak{S}_{p}$, giving $\sigma(s) \in S$. Conversely, suppose that $s, t \in S$ and

4 It can be verified directly that, in any topological group, the normalizer of a closed subgroup is closed. 
that $\sigma(s)=t$. Then $\mathbb{S}_{p}=\mathbb{S}_{t}=\sigma \mathcal{S}_{s} \sigma^{-1}=\sigma \mathbb{S}_{p} \sigma^{-1}$, showing that $\sigma \in \mathfrak{S}$. From this we derive the formula: if $E \subset S, \mathfrak{S}(E)=S \cap \mathbb{S}(E)$.

Since every element of $S$ is left fixed by $\mathcal{G}_{p}$ and $S$ is mapped into itself by $\mathfrak{Q}, \mathfrak{W} / \mathfrak{S}_{p}$ may be thought of as a compact Lie group acting on the space $S$. As a subspace of a completely regular space, $S$ is completely regular. No element of $S$ is left fixed by any element of $\mathfrak{W} / \mathbb{S}_{p}$ except the identity. By Theorem 3.3, there is a closed neighborhood $N_{1}$ of $p$ in $S$ and a closed subset $C$ of $N_{1}$ such that if $q \in N_{1}$, then $\left(\mathfrak{G} / \mathbb{S}_{p}\right)(q)=\mathfrak{Q}(q)$ intersects $C$ in exactly one point. Let $N=\mathbb{S}\left(N_{1}\right)$. Suppose that $r \in N$. Then $r=\sigma(q)$ for some $\sigma \in \mathbb{B}$ and $q \in N_{1}$, hence $\mathbb{B S}(r)=\mathbb{S}(q)$. Since $C \subset S, C \cap \mathbb{S}(r)=C \cap S \cap \mathbb{S}(q)$ $=C \cap \mathfrak{g}(q)$, which is a one element set. As a closed subset of the closed set $S, C$ is closed in $R$. We have now proved everything required except that $N$ is a closed neighborhood of $p$. We already know that it is closed by Lemma 2.1.

To show that $N$ is a neighborhood of $p$ we must show that $p \notin \mathrm{Cl}(\mathrm{R}-N)$ or since $p \in S$, that $p \notin S \cap \mathrm{Cl}(R-N)$. Using the formulas obtained in the first and second paragraphs of this proof, we have $R-N=\mathbb{R}-\mathbb{B}\left(N_{1}\right)=\$\left(\mathbb{R}-\mathbb{S}\left(N_{1}\right)\right)=\mathbb{S}\left(S-S \cap \circlearrowleft\left(N_{1}\right)\right)$ $=\mathbb{S}\left(S-\mathfrak{S}\left(N_{1}\right)\right)$. Using also the result of Lemma $2.2, S \cap C l(R-N)$ $=S \cap \mathrm{Cl}\left(\mathfrak{S}\left(S-\mathfrak{S}\left(N_{1}\right)\right)\right)=S \cap \mathbb{S}\left(\mathrm{Cl}\left(S-\mathfrak{E}\left(N_{1}\right)\right)\right)=\mathfrak{W}\left(\mathrm{Cl}\left(S-\mathfrak{S}\left(N_{1}\right)\right)\right)$ $=\mathrm{Cl}\left(\mathfrak{E}\left(S-\mathfrak{Q}\left(N_{1}\right)\right)\right)=\mathrm{Cl}\left(S-\mathfrak{W}\left(N_{1}\right)\right) \subset \mathrm{Cl}\left(S-N_{1}\right)$. Since $N_{1}$ is a neighborhood of $p$ in $S, p \notin \mathrm{Cl}\left(S-N_{1}\right)$, a fortiori $p \notin S \cap \mathrm{Cl}(R-N)$, and we are through.

3.5 COROLlaRY. Let (S) be a compact Lie group which acts on a completely regular space $R$. Let $p$ be a point of $R$ such that $\mathcal{G}_{p}$ is conjugate to $\mathbb{S}_{q}$ for all $q$ throughout a neighborhood of $p$. Then there exists a closed neighborhood $N$ of $p$ and a closed subset $C$ of $N$ such that the orbit of every point of $N$ has exactly one point in common with $C$.

Proof. Let $M$ be a closed neighborhood of $p$ such that $\mathbb{S}_{q}$ is conjugate to $\mathbb{S}_{p}$ if $q \in M$. The set $R^{\prime}=\mathbb{S}(M)$ is a closed subset of $R$. If $r \in R^{\prime}$, then $r=\sigma(q)$ where $\sigma \in \mathbb{S}^{\prime}$ and $q \in M$. Then $\mathbb{S}_{r}=\sigma \mathbb{S}_{q} \sigma^{-1}$ which is a conjugate of $\Theta_{p}$. We apply Theorem 3.4 to the space $R^{\prime}$ and observe that the set $N$ which is a closed neighborhood of $p$ in $R^{\prime}$ is also a closed neighborhood of $p$ in $R$. Likewise, the set $C$, being closed in $R^{\prime}$, is closed in $R$.

3.6 TheOREM. Let (S) be a compact Lie group which acts on a completely regular space $R$. Let $p$ be a point of $R$ such that $\mathbb{S}_{p}$ is conjugate to $\vec{B}_{q}$ for all $q$ throughout a neighborhood of $p$. Then there exists a closed neighborhood of $\mathbb{S}(p)$ which is fibered as a direct product by the orbits. 
Proof. We have only to observe that the set $C$ constructed in the proof of Theorem 3.4 satisfies the hypothesis of Theorem 2.3, while $\mathbb{S}(C)=\mathbb{S}(N)$ is a closed neighborhood of $\mathbb{S}(p)$.

4. Application to topological groups. We shall now use the previous results to investigate the nature of coset decompositions in a topological group. Theorem 4.1 is an immediate corollary of Theorem 3.6, while Theorem 4.2 extends this result to a broader class of subgroups.

4.1 THEOREM. Let $\Re$ be any topological group and let $\$$ be a subgroup which is a compact Lie group. Then there is a neighborhood of \&5 which is fibered as a direct product by the cosets of (S).

Proof. We may consider that the group $\mathbb{B}$ acts on the space $\Re$ by setting $\sigma(\tau)=\sigma \tau$ where $\sigma \in \mathbb{S}$ and $\tau \in \Re$. As a topological group, $\Re$ is a completely regular space. Since the orbits of points of $\Re$ are simply right cosets of $\mathbb{B}$, the theorem follows directly from Theorem 3.6. The same result for left coset decompositions follows by considering the anti-isomorphism $\sigma \rightarrow \sigma^{-1}$ of $\Re$ with itself.

4.2 TheOREM. Let $\Re$ be any topological group and let $\$$ be a closed subgroup of $\Re$ which is a covering group of a compact Lie group. Then there is a closed neighborhood of $\&$ which is fibered as a direct product by the cosets of 15 .

Proof. We shall construct a closed set $C$ in $\Re$ such that the mapping $\chi:(\sigma, \tau) \rightarrow \sigma \tau$ of $\$ \times C$ onto $\&(C)$ is a homeomorphism, and $(B)$ is a neighborhood of $B$.

Let $\Re$ be a discrete normal subgroup of $\&$ such that $\mathfrak{E}=\mathbb{S} / \Omega$ is a compact Lie group. Let $\psi$ be the natural map of $\mathbb{S}$ onto $\mathfrak{G}$. $\Omega$ is a discrete subgroup of $\Re$, hence we can form the homogeneous space $\Re / \Re$ of right cosets of $\Re$. Let $\phi$ be the natural map of $\Re$ onto $\Re / \Omega$. Since $\Re$ is discrete we can choose a closed neighborhood $S$ of $\epsilon$, the identity of $\Re$, such that $S S^{-1} \cap \Re=\{\epsilon\}$. It follows from this that $S \cap \rho S$ is void if $\rho \in \Re$ unless $\rho=\epsilon$. Let $\phi(S)=S_{1}$. Then $S_{1}$ is a neighborhood of $\phi(\epsilon)$ in $\Re / \Re$ and $\phi$ maps $S$ homeomorphically onto $S_{1}$. In fact, $\phi$ maps any set of the form $S \sigma(\sigma \in \Re)$ homeomorphically onto a neighborhood of $\phi(\sigma)$. Thus, since complete regularity is a local property, $\Re / \Re$ is a completely regular space.

Since $\Omega$ is a normal subgroup of $\mathbb{B}$, if $\sigma \in B$ and $\tau \in \Re$ we have $(\sigma \Omega)(\Omega \tau)=\sigma \Omega \tau=\Omega \sigma \tau$. It follows that the mapping $(\psi(\sigma), \phi(\tau))$ $\rightarrow \phi(\sigma \tau)$ of $\mathfrak{S} \times(\Re / \Re)$ onto $\Re / \Omega$ is well-defined. It is also continuous because of the continuity of the group operation and the definition of the topology $\mathfrak{S}$ and $\Re / \Re$. Thus the compact Lie group $\mathfrak{E}$ acts on the completely regular space $\Re / \Re$ under the convention $\psi(\sigma)(\phi(\tau))$ 
$=\phi(\sigma \tau)$. Furthermore, $\phi(\tau)=\psi(\sigma)(\phi(\tau))=\phi(\sigma \tau)$ only if $\sigma \in \Omega$; that is, if $\psi(\sigma)$ is the identity of $\mathfrak{S}$. By Theorem 3.3, we can choose a closed set $C_{0} \subset \Re / \Re$ which intersects each orbit of a closed neighborhood of $\mathfrak{W}(\phi(\epsilon))$ in just one point. We may assume $\phi(\epsilon) \in C_{0}$ (if not, we could replace $C_{0}$ by $\sigma\left(C_{0}\right)$ where $\sigma$ is an appropriate element of $\mathfrak{E}$ ). Let $C_{1}=S_{1} \cap C_{0}$. Then $C_{1}$ is a closed neighborhood of $\phi(\epsilon)$ in $C_{0}$. From the direct product representation of $\mathfrak{S}\left(C_{0}\right)$ (cf. Theorem 2.3) it follows that $\mathfrak{E}\left(C_{1}\right)$ is a closed neighborhood of $\mathfrak{W}(\phi(\epsilon))$.

Let $C=S \cap \phi^{-1}\left(C_{1}\right)$. Then $C$ is closed in $\Re$ and $\phi$ maps $C$ homeomorphically onto $C_{1}$. $C$ intersects each orbit of $B(C)$ exactly once. For suppose that $\rho \sigma=\tau$ where $\sigma, \tau \in C$ and $\rho \in(S$. Then $\phi(\tau)=\phi(\rho \sigma)$ $=\psi(\rho) \phi(\sigma)$ where $\phi(\tau), \phi(\sigma) \in C_{1}$. It follows that $\psi(\rho)$ is the identity of $\mathfrak{E}$ or that $\rho \in \Re$. Then $\tau \in S \cap \rho S$, where $\rho \in \Re$, but this set is void unless $\rho=\epsilon$. Hence $\sigma=\tau$. This implies that the mapping $\chi$ is one-toone. Being a restriction of the group mapping, $\chi$ is certainly continuous. To show that $\chi$ is a homeomorphism we must show. that it carries open sets into open sets. It is enough to show that all sufficiently small open sets containing a given point are carried into open sets.

Consider the composite mapping $(\sigma, \tau) \rightarrow(\psi(\sigma), \phi(\tau)) \rightarrow \psi(\sigma)(\phi(\tau))$

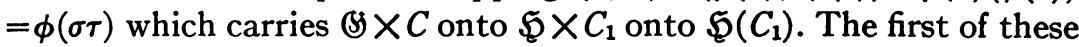
steps is a local homeomorphism and the second is a homeomorphism by Theorem 2.3. Since both steps carry open sets into open sets, the same is true of the composite which is simply $\phi \circ \chi$. Let $\sigma_{0} \in \mathbb{S}$ and $\tau_{0} \in C$ be fixed and let $V$ be an open neighborhood of $\sigma_{0} \tau_{0}$ so small that $\phi$ restricted to $V$ is one-to-one. Let $U$ be an open neighborhood of $\left(\sigma_{0}, \tau_{0}\right)$ so small that $\chi(U) \subset V$. Then $\chi(U)$ is open, for we know that $\phi \circ \chi(U)$ is open, and $\chi(U)=V \cap \phi^{-1}(\phi \circ \chi(U))$.

We have only to show that $B(C)$ is a closed neighborhood of $\$ S$ and the theorem will be proved. This follows immediately from the equation $\mathbb{S}(C)=\phi^{-1}\left(\mathfrak{S}\left(C_{1}\right)\right)$.

Society of Fellows, Harvard University 